\title{
The Influence of Estrous Cycle and Intrastriatal Estradiol on Sensorimotor Performance in the Female Rat
}

\author{
JILL B. BECKER, PETER J. SNYDER, MELISSA M. MILLER, \\ SARA A. WESTGATE AND MICHAEL J. JENUWINE \\ The University of Michigan, Psychology Department \\ and Neuroscience Laboratory Building, 1103 E. Huron, Ann Arbor, MI 48104-1687
}

Received 6 November 1986

\begin{abstract}
BECKER, J. B., P. J. SNYDER, M. M. MILLER, S. A. WESTGATE AND M. J. JENUWINE. The influence of estrous cycle and intrastriatal estradiol on sensorimotor performance in the female rat. PHARMACOL BIOCHEM BEHAV 27(1) $53-59,1987$. - The influence of estrous cycle and intrastriatal implants of 17beta-estradiol (17 $\beta$-estradiol). 17alphaestradiol (17 $\alpha$-estradiol) or cholesterol on the number of footfaults made by female rats traversing a narrow suspended beam was investigated. Female rats made fewer footfaults on estrus than on other days of the cycle. This was true when testing occurred during either the light or dark phase of the light:dark cycle. Intrastriatal implants of $30 \% 17 \beta$-estradiol for 6 hours resulted in a significant improvement in sensorimotor performance as soon as 4 hours after hormone implant and persisting for days. In contrast, intrastriatal implants of either $30 \% 17 \alpha$-estradiol or cholesterol had no influence on performance. The extent of hormone diffusion away from the implant cannula was minimal, and the resulting concentration of $17 \beta$-estradiol in the striatum was less than $10 \mathrm{pg} / \mathrm{mg}$. It is concluded that estradiol has a direct, stereospecific effect in the striatum that influences performance of a skilled motor act in the female rat.
\end{abstract}

Striatum Estrous cycle Estradiol Estrogen Sensorimotor performance Hormones and behavior

THE influence of estrous cycle on reproductive and nonreproductive behaviors in rodents has been well established. Female rats show estrous cycle dependent variation in sexual behavior [19], running wheel activity [3], and rotational behavior [7,35]. Many of these behaviors have been found to be associated with the increase in estrogen that triggers ovulation and the onset of behavioral estrus. For example, sexual behavior has been clearly demonstrated to depend on estrogen to activate neurons in the hypothalamus [31]. Estrogen is also implicated in the modulation of many nonreproductive behaviors. However, the neural site(s) of the estrogenic effects on non-reproductive behaviors are not well characterized.

One brain region that has been studied as a possible site of estrogen's effect on some non-reproductive behaviors is the striatum. This area of the brain has been implicated by studies reporting estrous cycle dependent variation in amphetamine-stimulated dopamine (DA) release from striatal tissue in vitro [5]. Furthermore, ovariectomy attenuates the AMPH-stimulated release of striatal DA and estrogen treatment reverses this effect of ovariectomy $[4,5]$. In addition, estrogen has been reported to potentiate striatal DA turnover [17] and induce an increase in the number of striatal DA receptors $[16,21,24,25]$.

Behavioral indices of striatal DA activity have also been found to be influenced by ovarian hormones. Rotational behavior, induced by electrical stimulation or amphetamine, shows estrous cycle dependent variation [7,35]. As was seen with striatal DA release, ovariectomy attenuates rotational behavior and estrogen treatment enhances this behavior [4, $13,25,35]$. Finally, intrastriatal estrogen implants have been shown to induce changes in postural deviation following systemic treatment with a dopamine agonist [26].

A potential problem with many of the previous studies investigating the effects of estrogen on DA-mediated behavior is that the behaviors are typically elicited with dopaminergic agonists or antagonists. This raises the possibility that the results are due to an interaction between estrogen and the drug used to induce the behavior. In the experiment reported here, we examine the influence of estrous cycle and intrastriatal estrogen implants on the accuracy of foot placement while traversing a suspended beam. Animals quickly learn to perform this task, and neither drugs nor food deprivation are necessary to induce the behavior. While this is a complex behavior that must involve activity in many brain regions in addition to the one under investigation here, our knowledge of basal ganglia function suggests that it should participate in the performance of this behavior (for a review see [29]). We present evidence that there is estrous cycle dependent variation in the performance of this sen- 

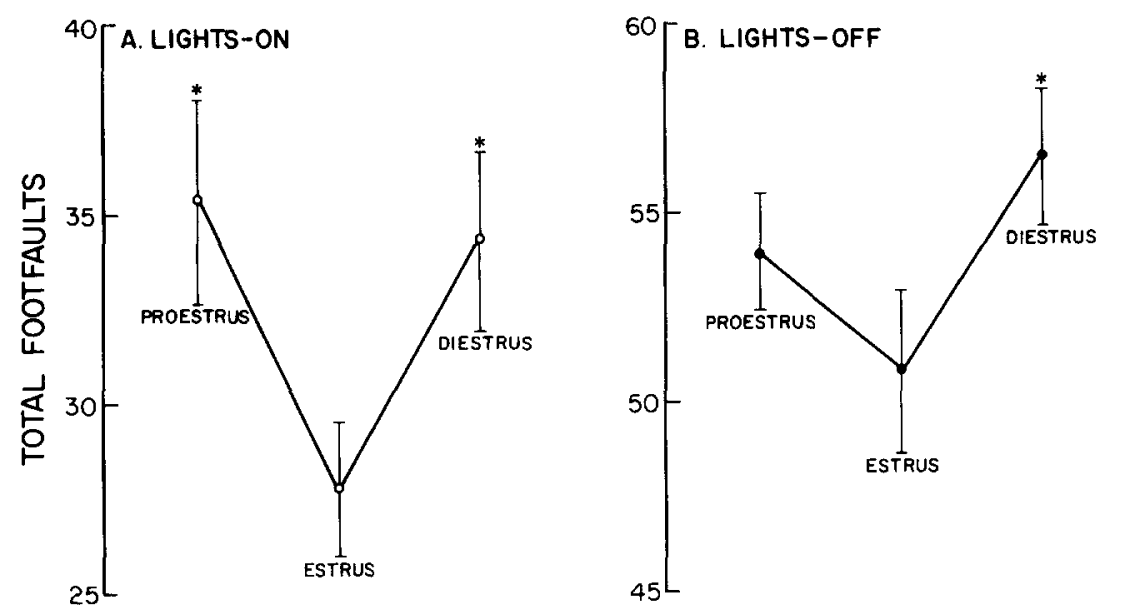

FIG. 1. The influence of estrous cycle and light/dark cycle on sensorimotor performance. Female rats were repeatedly tested 2 hours after lights-on (A) or two hours after lights-off (B). The mean number of footfaults made over 3 consecutive days during 4- or 5-day estrous cycles is graphed, bars indicate S.E.M. Note the different ranges in the scales for A and B. *-Significantly different from estrus $(p<0.05)$.

sorimotor task and that estrogen has specific effects on the striatum that influence performance of this task in ovariectomized female rats.

\section{METHOD}

\section{Experiment /}

This experiment was conducted to examine the influence of estrous cycle on sensorimotor performance in adult female Holtzman rats $(\mathrm{N}=9)$. Animals were individually housed in a room maintained on a 14:10, light:dark cycle with food and water freely available. Vaginal smears were taken daily to determine stage of estrous cycle.

The testing apparatus consisted of a wooden beam $5 \mathrm{ft}$ long and 0.75 in. wide, supported by two boxes, $4.5 \mathrm{ft}$ apart. At one end there was a start box, at the other end there was a partially enclosed dark box that the rat entered when it reached the end of the beam. The behavior was initially shaped using chocolate chip cookie mash for reward. After the rats had learned the task, the experimenter painted the rats' feet with a nontoxic tempura paint before each trial. The rat was placed in the start box behind a line marked on the beam. The experimenter timed the animal from when its front feet crossed the start line to the time it entered the dark box. Each rat was given two trials per day. For each rat there were 2 beams covered with plastic tape and permanently marked with the rat's number and trial number, A semipermanent record of each rat's performance was made by the tempura paint footprints left on the beams. One experimenter scored the animal's performance daily, recording the number of footprints that extended off the top of the beam onto the side (footfaults). After the beams were scored, the paint was washed off and the beams were reused. Both the experimenter conducting the behavioral trials and the one scoring the beams were unaware of the estrous cycle phase of the animals during the testing. All testing took place 4-6 hours after lights-on. Animals were followed over 2 weeks and only 4- or 5-day estrous cycles were used in the analysis.

At the conclusion of this series of tests, animals were transferred to a room maintained on a reversed light:dark cycle. After 4 weeks to adapt to the new lighting conditions, animals were retrained for 2 weeks and the procedure was repeated with testing taking place $2-4$ hours after lights-off, under red-light conditions. The estrous cycle was divided into days, each beginning with the onset of the dark period. The effect of time of day and estrous cycle on total footfaults (both trials combined) were analyzed by within-subjects analysis variance (repeated measures on time of day and estrous cycle phase).

\section{Experiment 2}

This experiment was conducted to determine whether the application of estrogen to the dorsal striatum could influence the ability of rats to traverse a suspended beam. Adult female Holtzman rats (housing conditions same as described above) were anesthetized with chloropent supplemented with methoxyflurane, and then animals were ovariectomized and also received bilateral implants of 22 gauge stainless steel guide cannulae aimed at the dorsal striatum. After 2 weeks for recovery, animals were trained on the sensorimotor task described in Experiment 1.

When performance on the task had stabilized, the effect of bilateral dorsal striatum application of: (1) $30 \%$ 17betaestradiol (17 $\beta$-estradiol; $70 \%$ cholesterol; $N=18$ ); (2) $30 \%$ 17alpha-estradiol ( $17 \alpha$-estradiol; $N=10$ ); or (3) cholesterol $(\mathrm{N}=17)$ on sensorimotor performance was examined. The crystalline injection/implant cannulae containing $30 \% \quad 17 \beta$ estradiol or $17 \alpha$-estradiol were prepared by mixing the appropriate amounts of estradiol with cholesterol, dissolving in absolute ethanol and evaporating to dryness. Steroids were tamped into 28 gauge stainless steel cannulae and the outside of the cannulae were cleaned with absolute ethanol.

On the day of the intrastriatal hormone injection, animals were first tested 2 hours after lights-on to determine baseline. Then each animal received cannulae containing one of the three steroid mixtures. (Stereotaxic coordinates for implants: $0.8 \mathrm{~mm}$ anterior and $2.5 \mathrm{~mm}$ lateral from Bregma, $4.0 \mathrm{~mm}$ ventral from top of skull.) Animals were retested $30-60 \mathrm{~min}$ and $4 \mathrm{hr}$ after insertion of the hormone-containing 
TABLE 1

INFLUENCE OF ESTROUS CYCLE AND LIGHT CYCLE ON TIME (SEC) TO TRAVERSE A SUSPENDED BEAM

\begin{tabular}{lllll}
$\begin{array}{l}\text { Lighting } \\
\text { Conditions } \\
\begin{array}{l}\text { During } \\
\text { Testing }\end{array}\end{array}$ & $(\mathrm{N})^{*}$ & Proestrus & Estrus & Diestrus \\
\hline & & & \multicolumn{3}{c}{ Day of Estrous Cycle } \\
Lights-on & $(18)$ & $2.2 \pm 0.1 \dagger$ & $2.5 \pm 0.2$ & $2.2 \pm 0.1$ \\
Lights-off & $(15)$ & $4.4 \pm 0.5$ & $3.8 \pm 0.4$ & $4.2 \pm 0.5$
\end{tabular}

*Nine female rats were repeatedly tested for 2 weeks. Those es trous cycles with vaginal smears clearly confirming proestrus, estrus and diestrus on 3 consecutive days were used in the analysis. All animals contributed at least 1 estrous cycle during each of the 2 lighting conditions.

$\uparrow$ Time in seconds. Mean \pm S.E.M.

cannulae. The implanted cannulae were removed after they had been in place for six hr and the stylettes replaced in the guide cannulae. Animals were tested again four hours after the cannulae had been removed (ten hr post-insertion), the next morning (two hr after light-on: $18 \mathrm{hr}$ after cannulae were removed), and then every 24 hours for six days.

As in Experiment 1, both the person conducting the behavioral tests and the person scoring the animals' performance were unaware of the animals' treatment group. The number of footfaults that each rat made after the steroid implants was expressed as a percent of the baseline performance on the implant day. The effect of hormone implants on sensorimotor performance was analyzed by between/within analysis of variance (steroid implanted $\times \%$ baseline over time).

After the conclusion of the experiment, the brains of these animals were prepared for histology, sectioned at 40 $\mu \mathrm{m}$ and stained with cresyl violet. Only data from animals with both implant cannulae positioned in the dorsal striatum were used in the analysis, all other were eliminated. (This resulted in the elimination of 2 rats from the $17 \beta$-estradiol group (see Fig. 3), 2 from the cholesterol group, and 3 rats from the $17 \alpha$-estradiol group.)

\section{Experiment 3}

This experiment was conducted to verify that the extent of spread of estradiol from the site of hormone implant in dorsal striatum was limited to the striatum. Ten mg of crystalline $30 \% 17 \beta$-estradiol were added to $1 \mathrm{mCi}$ of $17 \beta-(6,7-$ $\left[{ }^{3} \mathrm{H}\right] \mathrm{H}$ )-estradiol (60 Ci/mmole; Amersham Corp., Arlington Heights, IL) in ethanol and evaporated to dryness. The $\left[{ }^{3} \mathrm{H}\right]$ estradiol was tamped into 28 gauge stainless steel cannulae as described for Experiment 2. Rats prepared as described in Experiment 2 received bilateral implants of $\left[{ }^{3} \mathrm{H}\right]$ estradiol. Six hours later, the cannulae were removed and the animals were killed by decapitation.

The brains were rapidly removed and dissected into 1.5 $\mathrm{mm}$ slabs according to the method of Heffner et al. [23]. The slab(s) containing the injection cannula tips were identified and sliced at $1.5 \mathrm{~mm}$ intervals on a dorsal-ventral axis and again medial-laterally. The cube(s) at the tip of the cannula were pooled with corresponding cube(s) from the contralateral side. This tissue was designated as $<1.5 \mathrm{~mm}$ from the cannula tip. Only brains with cannulae that penetrated the corpus callosum and terminated in the dorsal striatum were

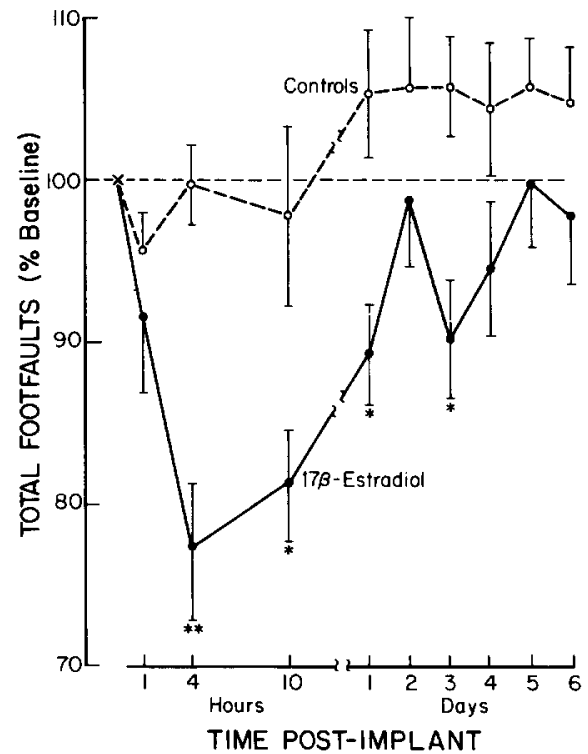

FIG. 2. The influence of intrastriatal implants of $30 \% 17 \beta$-estradiol on sensorimotor performance. The total number of footfaults is expressed as a percent of baseline performance. Controls received cannulae containing either $17 \alpha$-estradiol or cholesterol. ${ }^{* *}$-Four hours after hormone treatment, animals that had received $17 \beta$ estradiol showed a significant decrease in footfaults relative to controls $(p<0.005)$. *-Significantly different from controls $(p<0.05)$. Control substances did not differ from baseline or each other at any time (see text for details).

used. The tissue taken as $<1.5 \mathrm{~mm}$ was always exclusively striatum. Tissue cubes ventral, medial, and lateral (but not dorsal) to the ones just taken were pooled with those from the contralateral side $(1.5-3.0 \mathrm{~mm}$ from tip). The remaining tissue was designated as $>3.0 \mathrm{~mm}$ from tip. Tissue samples were placed in scintillation vials containing $1 \mathrm{ml}$ absolute ethanol and incubated at 50 degrees $C$ overnight. Five $\mathrm{ml}$ of ACS scintillation cocktail (Amersham Corp.) were added to each vial and samples were counted in a Beckman LS7500 liquid scintillation counter. Radioactivity was corrected for efficiency of the counter (35-40\%) to disintegrations/min and based on the specific activity of the $\left[{ }^{3} \mathrm{H}\right]$ estradiol mixture was expressed as picograms $\left[{ }^{3} \mathrm{H}\right]$ estradiol per $\mathrm{mg}$ tissue.

In order to examine the variability in the amount of $\left[{ }^{3} \mathrm{H}\right]$ estradiol that remained in each cannula, the cannulae after removal from the brain were placed in $500 \mu \mathrm{l}$ absolute ethanol and incubated at 50 degrees $C$ for $1 \mathrm{hr}$. Samples were counted in $5 \mathrm{ml}$ ACS scintillation cocktail as described above. The cannulae contained on the average $6.31 \mathrm{ng}$ estradiol $(n=18 ; 6.31 \pm 0.79 \mathrm{ng}$; range, 2.24-14.14 $\mathrm{ng}$ ).

\section{RESULTS}

\section{Experiment l}

There were significant effects of estrous cycle on the ability of female rats to accurately traverse a narrow beam, $\mathrm{F}(2,16)=4.82, p=0.022$ (Fig. 1). As is illustrated in Fig. 1A, when female rats were tested 4-6 hours after lights-on they made significantly fewer footfaults on estrus than on diestrus $(p<0.05)$ or proestrus $(p<0.05)$. This effect was observed for footfaults made with either the front or the back feet (data not shown). When the same animals were tested during 


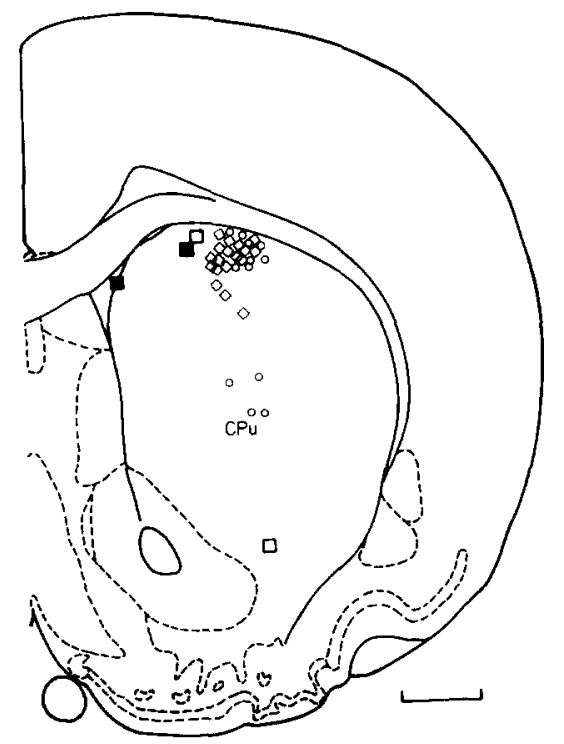

FIG. 3. Histological localization of cannulae tip placement for animals receiving implants of $17 \beta$-estradiol. Cannulae placements were collapsed along a rostral-caudal gradient between $0.2-1.2 \mathrm{~mm}$ rostral to Bregma [30], both left and right cannulae positions are illustrated on this diagram. The bar indicates $1.0 \mathrm{~mm}$. Open circles are used for animals that showed a 10-20\% improvement in performance 4-10 $\mathrm{hr}$ post-hormone application. The small open squares indicate animals that improved more than $20 \%$ (range $=20-47 \%$ ) during this same time period. The large squares indicate animals excluded from the experiment (see text for more details).

lights-off (under red-light conditions), they made significantly more footfaults overall than when testing occurred during lights-on, $F(1,8)=10.99, p=0.01$. However, there was still a significant effect of estrous cycle on this task. Fewer footfaults were made during estrus than during diestrus $(p<0.05)$. The effect of estrous cycle on sensorimotor performance was independent of the time the animals took to traverse the beam (runtime) as runtime did not vary with the estrous cycle (Table 1). However, when animals were tested under red-light conditions runtime was significantly greater than when testing occurred with the lights on $(p<0.001$; Table 1).

\section{Experiment 2}

There was no effect of cholesterol $(N=15)$ or $17 \alpha-$ estradiol $(\mathrm{N}=7)$ implants in the dorsal striatum on the total number of footfaults (percent baseline) made while traversing the beam. These groups also did not differ from each other, $F(1,20)=0.884$, so their results were pooled and designated the control group for subsequent analyses $(\mathrm{N}=22)$. In contrast, $17 \beta$-estradiol $(\mathrm{N}=16)$ implanted in the dorsal striatum resulted in a significant improvement in performance within $4 \mathrm{hr}$ of hormone administration (main effect of hormone: $F(1,36)=9.69, p=0.003$; hormone $\times$ time interaction: $F(8,288)=1.998, p=0.046$, Fig. 2$)$. The decrease in footfaults (percent baseline) persisted through testing $24 \mathrm{hr}$ postimplant, reoccurred 3 days post-implant, and then stabilized at the pre-implant performance rate. As was found for the estrous cycle, there was no effect of intrastriatal hormone application on runtime.

The placement of cannulae tips for all animals that received $17 \beta$-estradiol is illustrated in Fig. 3. All animals in-

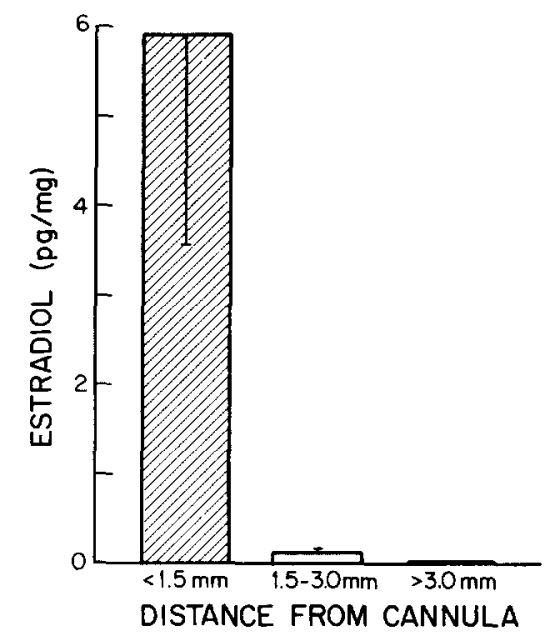

FIG. 4. Concentration of $17 \beta$-estradiol $(\mathrm{pg} / \mathrm{mg})$ in tissue after dorsal striatum implants. The hatched bar indicates concentration of estradiol in striatum $>1.5 \mathrm{~mm}$ from the cannula tip (S.E.M. indicated). Open bar indicates estradiol concentration in tissue $1.5-3.0 \mathrm{~mm}$ from cannula tip. Solid bar indicates concentration of $17 \beta$-estradiol $>3.0$ $\mathrm{mm}$ from cannula tip.

cluded in the analysis had both cannulae in the dorsal-medial striatum. The two animals excluded each had one cannula outside of the dorsal striatum. Interestingly, the animals that were excluded also did not experience a change in accuracy of foot placement following insertion of the hormone containing cannulae. The animal with both cannulae placements designated with a large solid square (Fig. 3) performed at $>100 \%$ of baseline from 1-10 hours post-hormone insertion and the animal with both cannulae placements designated with the large open squares (Fig. 3) performed at $>118 \%$ of baseline during this same time period.

\section{Experiment 3}

Six hours after cannulae had been implanted in the dorsal striatum only $0.52 \pm 0.15 \%$ (mean \pm S.E.M.) of the $\left[{ }^{3} \mathrm{H}\right]$ estradiol in the cannulae had diffused out of the cannulae. From this value, one can calculate that the dose delivered during the time that the cannula was implanted was approximately 33 picograms. In addition, approximately $90 \%$ of that amount $(89.4 \pm 1.7 \%)$ was found within the striatum, less than $1.5 \mathrm{~mm}$ from the cannula tip (Fig. 3). Therefore, the effective concentration of estradiol in the striatum achieved by the implants was $5.92 \pm 2.35 \mathrm{pg} / \mathrm{mg}$. Negligible quantities were found in the other areas of the brain.

\section{DISCUSSION}

The results indicate that estrogen can act in the dorsal striatum to facillitate the performance of a task requiring the integration of sensory and motor information. Female rats make fewer footfaults during estrus than on other days of the estrous cycle. The idea that this estrous cycle dependent intrastriatal implants of $30 \% 17 \beta$-estradiol also resulted in a significant improvement in sensorimotor performance. This effect of $17 \beta$-estradiol was due to a specific effect of estradiol in the striatum, as intrastriatal implants of $17 \beta$-estradiol or cholesterol did not influence the behavior and the spread of $17 \beta$-estradiol from the cannulae was localized within the striatum.

The finding that sensorimotor performance varied with 
the estrous cycle may be related to reports of estrous cycle dependent variation in other non-reproductive behaviors. In particular, there is estrous cycle dependent variation in locomotor activity, exploratory behavior, running wheel activity, and in sensory responsiveness (for review see [3]). Each of these behaviors has also been shown to increase following estrogen treatment [3]. In the experiments reported here, in addition to estrous cycle dependent variation in behavior, we also found that fewer footfaults were made during the testing that occurred with lights on than for testing during lights off. It is possible that this is related to the biorhythms in striatal metabolic and functional activity that have been reported (e.g., $[6,12,28,38])$. Alternatively, this could simply reflect a greater task difficulty under the redlight testing condition.

The time-course of the behavioral effect of endogenous estradiol fluctuations during the estrous cycle and the response to intrastriatal estradiol treatment are quite similar. During the estrous cycle, estradiol begins slowly rising during late diestrus, and then rises rapidly during the late morning of proestrus. The peak in estradiol concentrations in serum (for animals on a 14:10, light:dark cycle, lights-on at 6 a.m.) is found between 12-2 p.m. [11]. Since each phase of the estrous cycle was defined as beginning with the onset of the dark period, animals tested on proestrus were tested prior to the estrogen peak. Improvement in sensorimotor performance was found when animals were tested on estrus, 6 or 18 hours after the endogenous estrogen peak.

In comparison, the intrastriatal implants of estradiol were in place for 6 hours, and sensorimotor performance was facilitated within $4 \mathrm{hr}$ of initial hormone administration. The improvement in behavior persisted until $18 \mathrm{hr}$ after hormone removal ( $24 \mathrm{hr}$ after initial hormone administration). Performance had returned to baseline by the following test day ( 2 days after treatment). Therefore, the time course of the initial response to intrastriatal estrogen corresponds well with the time course of the effect seen during the estrous cycle. However, following intrastriatal estradiol there was also a second transient improvement in sensorimotor performance, 3 days after hormone treatment that does not have a correlate during the estrous cycle. There is also no time during the estrous cycle when the hormonal events are comparable. The increase in estrogen during the estrous cycle triggers a complex sequence of endocrine events that include (but are not limited to) ovulation as well as the secretion of progesterone and estrogen from the corpus luteum during diestrus [11]. Therefore, during the period of time when hormonal conditions are similar and comparisons are appropriate, the behavioral response to either endogenous ovarian hormones or intrastriatal estradiol is a facilitation of performance on our sensorimotor task.

The participation of the striatum in the modulation of sensorimotor function in the rat has been demonstrated in a number of different ways. For example, lesions of catecholamine neurons in the striatum with the neurotoxin 6-hydroxydopamine have been shown to disrupt skilled forepaw reaching in response to sensory cues $[18,36]$. In these experiments the animal can still perform the motor task and perceive the sensory cues but is unable to assimilate the sensory input fast enough to modify the ongoing motor behavior. In addition, it has been found that the rapid initiation of forepaw movement in response to a conditioned stimulus is dependent on the striatal catecholaminergic system with even small asymmetries inducing a decrement in response time [39]. In the cat, the influence of striatal lesions on walk- ing behavior has been studied, and in general lesions of the striatum result in very few gross motor deficits [41]. However, the authors report a "subjective lack of 'elegance,' 'slowness' and 'improvement' in the every day motor activities ..." following striatal lesions [41].

Another way to describe the role of the striatum in the integration of sensorimotor information has been suggested in a recent review of this topic [29]. Rather than directly controlling moyement, it is suggested that the basal ganglia functions as a sensory analyzer that filters and transduces incoming sensory information for use by motor systems [29]. Therefore, the role of the striatum in locomotion across a suspended beam would most likely be to facilitate the rapid, accurate placement of the rats' feet on the beam through analysis of sensory input. Our finding of hormonal influences in the striatum on footfaults made during locomotion across a suspended beam also argues that the striatum is critically involved in modulation of this task and that ovarian hormones can modulate functional activity in this neural system.

It is not known where or how estradiol is acting in the striatum to induce the behavioral response reported. Systemic estrogen is known to induce changes in the firing rates of substantia nigra DA neurons $[14,15]$ as well as in the activity of DA-sensitive neurons in the striatum [1]. However, whether the effect of estrogen on sensorimotor performance is a direct effect of estrogen on striatal DA neurons has not been examined. Estrogen could be acting on another population of neurons in the striatum to indirectly influence dopaminergic activity. Alternatively, this behavioral response to intrastriatal estrogen application could be independent of the striatal DA system.

In fact, how estrogen is able to influence neuronal activity in the striatum is unknown. The striatum contains very few (if any) genomic estrogen receptors [32]. The finding that neither $17 \alpha$-estradiol nor cholesterol were able to induce an altered behavioral response indicates that the effect of $17 \beta$ estradiol was not due to a nonspecific steroid effect. In addition, the response to $17 \beta$-estradiol was not mimicked by $17 \alpha$-estradiol, indicating that the response is stereospecific. These findings suggest that there may be an estrogen receptor, dissociable from the classical genome-activating steroid receptor, involved in the response to estrogen in the striatum.

The idea that the gonadal steroid hormones may influence cellular activity through more than one mechanism is not unique to striatum. For example, it has been known for some time that the response of the oocyte to steroids in amphibians is mediated by a cell surface receptor [37]. In mammals, the possibility of more than one type of estrogen receptor is suggested by reports of differential doseresponses of uterine cells to estrogen and its synthetic analogue diethylstilbesterol [22]. In addition, high-affinity membrane binding-sites for estrogen have been reported both in the rat uterus [33] and in the rat brain [40]. Furthermore, estrogen can stimulate DNA synthesis in uterine cells that lack nucleus-associated estrogen receptors [10]. All of this evidence suggests that steroid hormones may influence cellular activity, both in the brain and in the uterus, by more than one mcehanism. Elucidation of this alternative mechanism of hormone action may be important both for our understanding of reproductive function and for our understanding of hormonal influences on non-reproductive behaviors.

Finally, the finding that intrastriatal estrogen can influence an animal's sensorimotor behavior may also be of clinical relevance for the treatment of disorders of the basal gan- 
glia. For example, a single low dose of estradiol can increase lingual dyskinesia [8], while chronic estrogen treatment reduces tardive dyskinesia $[9,27]$. There is also clinical evidence that high estrogen concentrations can influence basal ganglia function in a small population of (normal) women, by inducing choreiform disorders during pregnancy (Chorea Graviderum) and/or in association with birth control pill use $[2,20,34]$. Elucidation of the mechanism(s) through which estrogen can modulate basal ganglia function may facilitate our understanding and treatment of these diseases.

\section{ACKNOWLEDGEMENTS}

This research was supported by a grant to J.B.B. from the National Science Foundation (No. BNS 84-11763). The authors would like to thank C. J. Moore and M. Kalafut for their technical assistance and Drs. T. E. Robinson and E. S. Valenstein for their helpful comments on an earlier version of this manuscript.

\section{REFERENCES}

1. Arnauld, E., B. Dufy, M. Pestre and J. D. Vincent. Effects of estrogens on the responses of caudate neurons to microiontophoretically applied dopamine. Neurosci Lett 21: 325-331, 1981.

2. Barber, P. V., A. G. Arnold and G. Evans. Recurrent hormone dependent chorea: effects of oestrogens and progestagens. Clin Endocrinol 5: 291-293, 1973.

3. Beatty, W. W. Gonadal hormones and sex differences in nonreproductive behaviors in rodents: organizational and activational influences. Horm Behav 12: 112-163, 1979.

4. Becker, J. B. and M. E. Beer. The influence of estrogen on nigrostriatal dopamine activity: behavioral and neurochemical evidence for both pre- and postsynaptic components. Behav Brain Res 19: 27-33, 1986.

5. Becker, J. B. and V. D. Ramirez. Sex differrences in the amphetamine-stimulated release of catecholamines from rat striatal tissue in vitro. Brain Res 204: 361-372, 1981.

6. Becker, J. B., M. E. Beer and T. E. Robinson. Striatal dopamine release stimulated by amphetamine or potassium: influence of ovarian hormones and the light-dark cycle. Brain Res 311: 157-160, 1984.

7. Becker, J. B., T. E. Robinson and K. A. Lorenz. Sex differences and estrous cycle variations in amphetamine-elicited rotational behavior. Eur J Pharmacol 80: 65-72, 1982.

8. Bédard, P. J., R. Boucher, M. Daigle and T. DiPaolo. Physiological doses of estradiol can increase lingual dyskinesia and cerebrospinal fluid homovanillic acid in monkeys. Neurosci Lett 58: 327-331, 1985.

9. Bédard, P. J., R. Boucher, M. Daigle and T. DiPaolo. Similar effect of estradiol and domperidone on lingual dyskinesia in monkeys. Psychoneuroendocrinology 9: 375-379, 1984.

10. Bigsby, R. M. and G. R. Cunha. Estrogen stimulation of deoxyribonucleic acid synthesis in uterine epithelial cells which lack estrogen receptors. Endocrinology 119: 390-396, 1986.

11. Butcher, R. L., W. E. Collins and N. W. Fugo. Plasma concentrations of LH, FSH, prolactin, progesterone and estradiol-17 $\beta$ throughout the 4-day estrous cycle of the rat. Endocrinology 94: 1704-1708, 1974.

12. Cahill, A. L. and C. F. Ehret. Circadian variations in the activity of tyrosine hydroxylase, tyrosine aminotransferase, and tryptophan hydroxylase: relationship to catecholamine metabolism. J Neurochem 37: 1109-1115, 1981.

13. Camp, D. M., J. B. Becker and T. E. Robinson. Sex differences in the effects of gonadectomy on amphetamine-induced rotational behavior in rats. Behav Neural Biol 46: 491-495, 1986.

14. Chiodo, L. A. and A. R. Caggiula. Alterations in basal firing rate and autoreceptor sensitivity of dopamine neurons in the substantia nigra following acute and extended exposure to estrogen. Eur J Pharmac ol 67: 165-166, 1980.

15. Chiodo, L. A., W. M. Glazer and B. S. Bunney. Midbrain dopamine neurons: electrophysiological studies on the acute effects of estrogen. In: Dopaminergic Systems and Their Regulation, edited by R. G. Woodruff. New York: McMillan Press Inc., 1986.
16. DiPaolo, T., P. J. Bédard, A. Dupont, P. Poyet and F. Labrie. Effects of estradiol on intact and denervated striatal dopamine receptors and on dopamine levels: a biochemical and behavioral study. Can J Physiol Pharmacol 60: 350-357, 1982.

17. DiPaolo, T., C. Rouillard and P. J. Bédard. $17 \beta$-Estradiol at a physiological dose acutely increases dopamine turnover in rat brain. Eur J Pharmacol 117: 197-203, 1985.

18. Evenden, J. L. and T. W. Robbins. Effects of unilateral 6-hydroxydopamine lesions of the caudate-putamen on skilled forepaw use in the rat. Behav Brain Res 14: 61-68, 1984.

19. Feder, H. H. Estrous cyclicity in mammals. In: Neuroendocrinology of Reproduction: Physiology and Behavior, edited by $\mathrm{N}$. T. Adler. New York: Plenum Press, 1981, pp. 279-348.

20. Gamboa, E. T., G. Isaacs and D. Harter. Chorea associated with oral contraceptive therapy. Arch Neurol 25: 112-114, 1971.

21. Gordon, J. H. and K. O. Perry. Pre- and postsynaptic neurochemical alterations following estrogen-induced striatal dopamine hypo- and hypersensitivity. Brain Res Bull 10: 425428, 1983.

22. Grunert, G., M. Porcia and A. N. Tchernitchin. Differential potency of oestradiol-17\% and diethylstilboestrol on separate groups of response in the rat uterus. J Endocrinol 110: 103-114, 1986.

23. Heffner, T. G., J. A. Hartman and L. S. Seiden. A rapid method for the regional dissection of the rat brain. Pharmacol Biochem Behav 13: 453-456, 1980.

24. Hruska, R. E., L. M. Ludmer and E. K. Silbergeld. Characterization of the striatal dopamine receptor supersensitivity produced by estrogen treatment of male rats. Neuropharmacology 19: 923-926, 1980.

25. Hruska, R. E. and E. K. Silbergeld. Increased dopamine receptor sensitivity after estrogen treatment using the rat rotation model. Science 208: 1466-1468, 1980.

26. Joyce, J. N, and C. Van Hartesveldt. Estradiol application to one striatum produces postural deviation to systemic apomorphine. Pharmacol Biochem Behav 20: 575-581, 1984.

27. Koller, W. C., A. Barr and N. Biary. Estrogen treatment of dyskinetic disorders. Neurology 32: 547-550, 1982.

28. Lemmer, $R$. and $T$. Berger. Diurnal rhythm in the central dopamine turnover in the rat. Naunyn Schmiedebergs Arch Pharmacol 303: 257-261, 1978.

29. Lidsky, T. I., C. Manetto and J. S. Schneider. Consideration of sensory factors involved in motor functions of the basal ganglia. Brain Res Rev 9: 133-146, 1985.

30. Paxinos, G. and C. Watson. The Rat Brain in Stereotaxic Coordinates. New York: Academic Press Inc., 1982.

31. Pfaff, D. W. Estrogens and Brain Function. Neural Analysis of Hormone-Controlled Mammalian Reproductive Behavior. New York: Springer-Verlag, 1980.

32. Pfaff, D. W. and M. Keiner. Atlas of estradiol-concentrating cells in the central nervous system of the female rat. Comp Neurol 151: 121-158, 1973.

33. Pietras, R. J. and C. M. Szego. Estrogen receptors in uterine plasma membranes. J Steroid Biochem 11: 1471-1483, 1979. 
34. Riddoch, D., M. Jefferson and E. R. Bickerstaff. Chorea and oral contraceptives. Br Med J 4: 217-218, 1971.

35. Robinson, T. E., D. M. Camp, D. S. Jacknow and J. B. Becker. Sex differences and estrous cycle dependent variation in rotational behavior elicited by electrical stimulation of the mesostriatal dopamine system. Behav Brain Res 6: 273-287, 1982.

36. Sabol, K. E., D. B. Neill, S. A. Wages, W. H. Church and J. B. Justice. Dopamine depletion in a striatal subregion disrupts performance of a skilled motor task in the rat. Brain Res 335: 33-43, 1985.

37. Sadler, S. E. and J. L. Maller. Plasma membrane steroid hormone receptors. In: The Receptors, Vol I, edited by P. M. Conn. New York: Academic Press, Inc., 1984, pp. 431-463.
38. Simon, M. L. and R. George. Diurnal variation in plasma corticosterone and growth hormone as correlated with regional variations in norepineprhine, dopamine and serotonin content of rat brain. Neuroendocrinology 17: 125-138, 1975.

39. Spirduso, W. W., P. E. Gilliam, T. Schallert, M. Upchurch, D. M. Vaughn and R. E. Wilcox. Reactive capacity: A sensitive behavioral marker of movement initiation and nigrostriatal dopamine function. Brain Res 335: 45-54, 1985.

40. Towle, A. C. and P. Y. Sze. Steroid binding to synaptic membranes: differential binding of glucocorticoids and gonadal steroids. J Steroid Biochem 18: 135-143, 1983.

41. Villablanca, J. R., R. J. Marcus and C. E. Olmstead. Effects of caudate nuclei or frontal cortical ablations in cats. I. Neurology and gross behavior. Exp Neurol 52: 389-420, 1976. 